\title{
DISCUSIONES ACTUALES EN TORNO A LA \\ LUSOFONÍA: PANORAMA DE LOS ESTUDIOS SOBRE POLITICA INTERNACIONAL DEL PORTUGUÉS
}

\author{
CURRENT DISCUSSIONS ON LUSOPHONY: AN OVERVIEW \\ OF THE INTERNATIONAL POLICY STUDIES OF PORTUGUESE
}

\section{María Florencia Rizzo*}

\section{RESUMEN}

La lengua portuguesa tiene un peso considerable en el actual escenario global: está presente en América, África, Europa, Asia y cuenta con más de doscientos cincuenta millones de hablantes como primera o segunda lengua; a nivel mundial, ocupa el séptimo lugar (CALVET y CALVET, 2013). La gestión internacional de esta lengua ha sido conducida históricamente por organismos vinculados a los gobiernos de Portugal y de Brasil, mientras que los demás territorios nacionales que integran la Comunidad de Países de Lengua Portuguesa han desempeñado un papel modesto (OLIVEIRA y DORNELLES, 2007). Por su posición histórica de metrópoli, Portugal se ha propuesto como país que "legítimamente" ocuparía el centro del área idiomática; sin embargo, en los últimos años Brasil, aprovechando su lugar preponderante en la región sudamericana, ha adquirido un creciente protagonismo en la gestión internacional de la lengua. Este artículo realiza una revisión bibliográfica que recorre una serie de ejes de análisis que atraviesan las investigaciones actuales sobre lusofonía, entendida como política de área idiomática del portugués destinada a gestionar la lengua compartida en el espacio que comprende a la Comunidad de Países de Lengua Portuguesa. En primer lugar, nos centraremos en la evaluación que los estudios considerados realizan sobre la política internacional del portugués, atendiendo especialmente a la dinámica de fuerzas entre acciones nacionales y multilaterales así como al papel de las integraciones regionales en dicha dinámica. En segundo lugar, presentaremos distintas perspectivas teóricas sobre el concepto de "lusofonía" y revisaremos dos problemáticas que atraviesan las políticas actuales: la interpretación en clave neocolonizadora -o su rechazo- de los proyectos y las acciones de gestión internacional del portugués o del modo en que son percibidos; y la identificación de tendencias homogeneizantes en determinadas políticas lingüísticas que buscan imponer una realidad dominante.

Palabras-clave: lusofonía; política internacional del portugués; Comunidad de Países de Lengua Portuguesa.

\section{ABSTRACT}

The Portuguese language has considerable weight in the current global scene: it is present in America, Africa, Europe, Asia and has more than two hundred and fifty million speakers as first or second language. On a world-wide scale, it occupies the seventh place (CALVET and CALVET, 2013). The international management of this language has historically been

\footnotetext{
*CONICET/Universidad Nacional de San Martín, Argentina. rizzoflorencia@gmail.com
} 
conducted by organizations linked to the governments of Portugal and Brazil, while the other national territories that make up the Community of Portuguese Language Countries have played a modest role (OLIVEIRA and DORNELLES, 2007). Due to its historical position as a metropolis, Portugal has proposed itself as a country that would "legitimately" occupy the center of the idiomatic area. However, in recent years Brazil, taking advantage of its preponderant place in the South American region, has acquired a growing role in the international management of language. This article carries out a bibliographical review that runs through a series of themes of analysis that straddle current research on Lusophony, understood as a language policy of Portuguese aimed at managing the language shared in the space that includes the Community of Portuguese Language Countries. Firstly, we will focus on the evaluation that the considered studies make on the international policies of Portuguese, paying particular attention to the dynamics of forces between national and multilateral actions as well as the role of regional integrations in the aforementioned dynamics. Secondly, we will present different theoretical perspectives on the concept of "Lusophony" and we will review two problems that current policies go through: the interpretation from a (neo)colonizing perspective -or its rejection- of projects and international management actions of the Portuguese or the way they are perceived; and the identification of homogenizing tendencies in certain language policies that seek to impose a dominant reality.

Keywords: Lusophony; international policy of Portuguese; Community of Portuguese Language Countries.

\section{INTRODUCCIÓN}

El reordenamiento geográfico y político que implica la formación de bloques regionales, en constante evolución, ha tenido un fuerte impacto sobre las funciones de los Estados nacionales, donde entra en juego la tensión entre lo local, lo nacional, lo regional y lo global. En lo que concierne al ámbito de las lenguas, por un lado, aquellos han tenido que gestionar la diversidad lingüística dentro de sus territorios, donde conviven distintas variedades; por el otro, sus tareas han trascendido las fronteras nacionales, en especial, en lo que respecta al desarrollo de políticas de áreas idiomáticas lideradas por los países que cuentan con mayor peso político y económico (ARNOUX, 2011). En el caso del ámbito lusófono, la gestión de la lengua ha sido conducida históricamente por organismos vinculados a los gobiernos de Portugal y de Brasil, mientras que los demás territorios nacionales que integran la llamada Comunidad de Países de Lengua Portuguesa (CPLP) han desempeñado un papel modesto (OLIVEIRA y DORNELLES, 2007). De modo que se ha producido desde muy pronto una situación de bicentrismo normativo ya que los Países Africanos de Lengua Oficial Portuguesa (PALOP) han estado hasta hace poco tiempo bajo la influencia de la norma lisboeta (LAGARES, 2016). Así, por un lado, Portugal se propone como país que "legítimamente", por su posición 
histórica de metrópoli, ocuparía el centro del área idiomática; mientras que Brasil, aprovechando su lugar preponderante en la región sudamericana, en los últimos años ha adquirido un mayor liderazgo en la gestión de la llamada "lusofonía".

La lengua portuguesa tiene un peso considerable en el escenario global: está presente en América, África, Europa y Asia y cuenta con más de doscientos cincuenta millones de hablantes como primera o segunda lengua. Es idioma oficial en los nueve Estados que integran la CPLP: Angola, Brasil, Cabo Verde, GuineaBissau, Mozambique, Portugal, Santo Tomé y Príncipe, Timor del Este y Guinea Ecuatorial, país recientemente incorporado ${ }^{1}$; también en la región china de Macao donde es cooficial junto al mandarín. A nivel mundial, de acuerdo con un barómetro de las lenguas que contempla factores tales como el número de hablantes, el estatuto de la lengua y la función vehicular, el portugués ocupa el séptimo lugar (CALVET y CALVET, 2013).

El propósito de este artículo es realizar un recorrido por un conjunto de problemáticas y discusiones que atraviesan los estudios actuales sobre lusofonía, entendida aquí como política de área idiomática del portugués destinada a gestionar la lengua compartida en determinado espacio el cual, en líneas generales, coincide con el que reúne a los países que componen la CPLP. Se trata de un trabajo de revisión crítica de bibliografía que pretende, así, a partir del relevamiento de algunos ejes de análisis abordados por investigaciones producidas en los últimos años, realizar una contribución al ámbito de los estudios glotopolíticos (ARNOUX, 2000, 2014; ARNOUX y DEL VALLE, 2010; DEL VALLE, 2017), atentos a la articulación entre las intervenciones en el espacio público del lenguaje y las ideologías lingüísticas asociadas a ellas, a la comprensión de sus implicancias sociales y a la interpretación de su sentido histórico, considerando con particular atención espacios donde se expresan diferencias, tensiones y disputas. De ahí que nuestro mayor interés se oriente hacia trabajos que interpelan la dimensión ideológica de las políticas lingüísticas destinadas a la internacionalización del portugués.

\section{CONSIDERACIONES METODOLÓGICAS}

Consideramos que los estudios incluidos en esta investigación pueden inscribirse, a grandes rasgos, en el amplio campo de la

1. La incorporación de Guinea Ecuatorial como Estado miembro en 2014 fue fuertemente cuestionada debido a las numerosas denuncias que recaían sobre el gobierno por violación a los derechos humanos (LEVISKI, 2015; SEVERO, 2016; BAGNO, 2018). 
Glotopolítica ${ }^{2}$ lo cual supuso dejar de lado numerosos análisis desde otros enfoques dentro de los que se encuentran los estudios culturales y literarios, las ciencias políticas o las relaciones internacionales. En cuanto a los materiales de archivo relevados, la selección incluyó mayormente artículos de revistas académicas y libros especializados o capítulos publicados en los últimos diez años.

La circulación del término lusofonía ha tenido un peso desigual en el mundo lusohablante (CASTRO, 2008; LAGARES, 2016). Según Faraco (2016, p. 318$319)$, todo indica que la noción comenzó a tener una mayor difusión en el contexto portugués años después de la descolonización, entre fines de la década de 1980 y principios de la de 1990, en el marco del proceso de integración a la Unión Europea y de la recomposición de los vínculos con los países africanos y con Brasil, lo cual le permitiría a Portugal ubicarse como interlocutor privilegiado entre estos y Europa. En Brasil, en cambio, la repercusión del término ha sido mucho menor; en el caso de los países africanos, con una independencia joven, los emprendimientos de la lusofonía se han visto con desconfianza ya que se percibe en ellos un proyecto político unificador y de base neocolonial (COUTO, 2009; SEVERO, 2016; FARACO, 2016).

Aunque se harán algunas referencias específicas, los trabajos que son objeto de este artículo no abordan la lusofonía en sentido amplio como comunidad de lengua portuguesa en el mundo ni como forma primordialmente identitaria (como en CRISTÓVÃO, 2008; MARTINS, 2014; HANNA, BRITO y BASTOS, 2010; BRITO y BASTOS, 2011) sino como política lingüística del área idiomática del portugués. Atendiendo a una perspectiva glotopolítica, la mayoría de los estudios relevados se distancia de la noción de lusofonía entendida como espacio lingüístico no conflictivo, como unidad armónica de la lengua portuguesa. En cambio, la considera dentro de un proceso histórico de construcción política de esta lengua atravesado por ciertas pugnas y tensiones que remiten a cuestiones sensibles de la historia de los países involucrados, especialmente a las vinculadas con el impacto de los procesos de (des)colonización.

En concordancia con lo anterior, hemos planteado una serie de interrogantes como disparadores que orientaron la lectura de los textos: ¿Cómo es concebida la

2. Esta decisión se apoya en que la Glotopolítica incluye lo que tradicionalmente se designa con el sintagma política lingiística y amplía su alcance: considera diversas acciones, no solo desde instancias estatales, sino también desde esferas no oficiales, emprendidas por ciertas instituciones o sectores de la sociedad civil. Los materiales discursivos donde pueden estudiarse estas intervenciones también son variados: instrumentos lingüísticos, manuales de estilo, ensayos, artículos periodísticos, manuales escolares, documentos institucionales, entre muchos otros. Para profundizar el enfoque glotopolítico y su relación con la política lingüística, ver Arnoux (2014). 
lusofonía? ¿Qué evaluaciones surgen de los análisis sobre las políticas lingüísticas nacionales y transnacionales respecto del portugués, implementadas hasta el momento, y de la relación -en términos de equilibrio, complementariedad, tensión, competencia, etc.- entre ambas? ¿Qué lugar ocupan en dichas políticas las otras lenguas que conviven con el portugués? ¿Cuáles son las propuestas para una adecuada gestión internacional de la lengua? ¿Qué papel desempeña en las cuestiones anteriores la tensión histórica entre (ex)colonizador y (ex)colonizado? Siguiendo estas preguntas, hemos organizado el trabajo en dos grandes partes compuestas, a su vez, por distintos apartados estrechamente relacionados entre sí: en primer lugar, nos centraremos en la evaluación que los estudios considerados realizan sobre la política internacional del portugués, atendiendo especialmente a la dinámica de fuerzas entre acciones nacionales y multilaterales así como al papel de las integraciones regionales en dicha dinámica; en segundo lugar, presentaremos distintas perspectivas teóricas sobre el concepto de "lusofonía" y revisaremos dos problemáticas que atraviesan las políticas actuales: la interpretación en clave (neo) colonizadora -o su rechazo- de los proyectos y las acciones de gestión internacional del portugués o del modo en que son percibidos; y la identificación de tendencias homogeneizantes en determinadas políticas lingüísticas que buscan imponer una realidad dominante.

\section{LA POLÍTICA INTERNACIONAL DEL PORTUGUÉS}

El área idiomática de la lusofonía se caracteriza por la ausencia de una única entidad reguladora fuerte $y$, en consecuencia, de instrumentos lingüísticos que asuman el papel representativo de todas las variedades como un todo (CORREIA y FERREIRA, 2013, p. 318). De acuerdo con Lagares (2018, p. 196-197), la falta de un organismo legitimado históricamente -como ocurre con la Real Academia Española en el caso del ámbito hispánico- favoreció una gestión normativa del portugués enmarcada en las acciones legislativas de los Estados nacionales. Entre ellos, concentran la política internacional del portugués los dos países donde esta lengua está consolidada político-nacionalmente (SILVA, 2010a): Portugal y Brasil. En lo que concierne a los países africanos de habla portuguesa y Timor Oriental, siguiendo a Silva (2013), estos no han mostrado políticas internacionales relevantes para la difusión de la lengua portuguesa, excepto a través de la CPLP.

Además de las gestiones llevadas a cabo desde esferas nacionales, principalmente a través de la actuación de los ministerios de educación y de relaciones exteriores brasileños y portugueses, se han desarrollado iniciativas 
multilaterales provenientes del conjunto de Estados que tienen el portugués como lengua oficial, aunque siempre con mayor peso de los centros mencionados: entre ellas, se destaca el Acuerdo Ortográfico de la Lengua Portuguesa (AOLP), la constitución de la CPLP y, como parte de esta entidad, la creación del Instituto de Investigación de Lengua Portuguesa (IILP). En términos generales, tanto las iniciativas como los discursos que las difunden provienen mayormente de organismos estatales, nacionales e internacionales. Estos rasgos marcan a simple vista diferencias importantes respecto de otras áreas idiomáticas como la hispanofonía donde, como señalamos, las academias de la lengua, en especial la Real Academia Española, tienen una visibilidad y un peso considerable, y donde los materiales discursivos de promoción internacional son, en gran medida, resultado de prácticas de divulgación que cuentan con la participación de figuras públicas que actúan como una suerte de representantes del panbispanismo, (tal es término elegido por sus ideólogos); nos referimos a eventos de gran repercusión mediática como los congresos internacionales de la lengua española ${ }^{3}$ o notas y entrevistas que muchos académicos brindan en importantes medios de comunicación. ${ }^{4}$

\subsection{La lusofonía: entre iniciativas nacionales y multilaterales}

Oliveira y Dornelles (2007) y Faraco $(2010,2016)$ coinciden en que la tendencia de la política lingüística internacional desplegada por parte de los dos Estados donde el portugués es lengua mayoritaria y hegemónica, esto es, Portugal y Brasil, ha sido hacia acciones divergentes; de modo que se han desplegado políticas nacionales aisladas, las cuales, en varias ocasiones, han entrado en tensión. Un ejemplo de esto se evidencia en el análisis que realiza Zoppi-Fontana (2012) en torno a la creación en 2006 del Día Nacional de la Lengua Portuguesa en Brasil, acontecimiento que tomó distancia de la fecha que había sido designada en 2004 para celebrar la lengua portuguesa en el mundo lusófono en conmemoración por la muerte de Camões. ${ }^{5}$ Esta iniciativa brasileña se inscribiría, siguiendo la propuesta de Castro (2008), quien identifica dos pulsiones en el mundo de la lengua portuguesa, la separatista y la unificadora, dentro de la primera tendencia; mientras que el

3. Sobre estos encuentros y la política panbispánica que difunden, ver Rizzo (2014).

4. Para un estudio de las dinámicas normativas de la lusofonía y de la hispanofonía, ver Lagares (2016, 2018). Asimismo, Faraco (2010), Lagares (2013) y Oliveira (2013b) realizan comparaciones entre distintas fonías.

5. Continuando con el estudio de Zoppi-Fontana (2012), esa asociación de la lengua a determinado territorio nacional fue posteriormente dislocada por el funcionamiento de una nueva fecha de celebración: el Día de la Lengua Portuguesa y de la Cultura de la CPLP, establecido en 2009, determinando este espacio supranacional como territorio simbólico de referencia. 
proyecto de la lusofonía que se plasma, por ejemplo, en la CPLP, se orienta hacia la segunda.

Este organismo fue creado en 1996 con la finalidad de desarrollar una política lingüística multilateral de los países con lengua oficial portuguesa en torno a tres objetivos generales: la articulación político-diplomática entre sus miembros; la cooperación económica, social, cultural, jurídica y técnico-científica; y la promoción y difusión de la lengua portuguesa. En términos de Faraco (2016, p. 326), esta entidad supranacional ha materializado institucionalmente la lusofonía; sin embargo, el autor señala la conveniencia de llevar adelante acciones convergentes de gestión y promoción coordinadas colectivamente en un espacio institucional multilateral, sin perjuicio de otros programas en el ámbito nacional (FARACO, 2016, p. 353).

Para Oliveira (2013b) la CPLP contiene desde su concepción la idea de gestión transnacional o global del portugués pero aún está lejos de cumplir ese propósito ya que se encuentra a medio camino entre una realidad puramente nacional y otra, incipiente, que se acerca a una etapa multinacional. Desde esta perspectiva, para internacionalizar la lengua "precisamos internacionalizar a sua gestão, construindo de maneira conjunta a sua cadeia tecnológica e a coordenação diplomática da sua negociação global, reconhecendo a oportunidade de pensa-la e trata-la como LÍNGUA POLICÊNTRICA" (OLIVEIRA, 2013b, p. 432).

En una dirección similar, Severo (2016, p. 1329) argumenta que la CPLP se presenta como una comunidad transnacional, pero "esse transnacionalismo não tem sido suficiente para colocar em xeque os limites do Estado-nação e das fronteiras que definem o que conta como "língua portuguesa'". De acuerdo con la autora, la tensión entre Estado-nación y comunidad transnacional resuena en las políticas ambivalentes de la CPLP y del IILP. La ideología nacionalista se evidencia en acciones del gobierno brasileño, por ejemplo, en la exigencia de pruebas de competencia en lengua portuguesa (Certificação de Proficiência em Língua Portuguesa para Estrangeiros, Celpe-Bras) para países miembros de la CPLP (cf. DINIZ y BIZÓN, 2015), pero también por parte del Estado portugués en defensa de la lusitanidad.

En lo que concierne a la pregunta explícita por los agentes a cargo de la gestión internacional del portugués, a partir de una comparación de datos económicos de Brasil y de Portugal, Bagno (2009) y Bagno y Carvalho (2015) plantean que el país sudamericano debería estar al frente de aquella empresa. Así, al argumentar la oposición a la pretensión de liderazgo de Portugal aplican la misma lógica que critican y se distancian, de este modo, de posturas como las comentadas en los párrafos anteriores que se manifiestan, de modo más o menos 
explícito y con distintos matices, a favor de una gestión compartida. Situado, en cambio, en el contexto portugués, Reis (2010) postula que una política lingüística de internacionalización de la lengua portuguesa debe basarse en "alianzas bien pensadas" dentro del territorio de Portugal y entre este país y los restantes de la CPLP a los que debe convocar, con lo cual otorga un mayor protagonismo al país europeo.

Entre los trabajos que se ocupan de la constitución y las políticas de la CPLP, un conjunto se detiene en el IILP, que ha sido identificado como el primer instrumento institucional de aquella entidad (CARVALHO, 2012). En líneas generales, los autores coinciden en que el IILP se mantuvo en una suerte de letargo durante el primer período, desde su creación en 1999 hasta alrededor del 2010 cuando comenzó a tener un papel más activo; este momento coincidió con el mandato del lingüista brasileño Gilvan Müller de Oliveira, quien ejerció la dirección ejecutiva hasta 2014. Articulando la experiencia en la gestión y la labor de investigación, Oliveira (2015) estudia el funcionamiento del IILP y propone una periodización de su historia. Para el autor se trata de una institución original que se distingue de otras organizaciones de política lingüística en la medida en que concibe la lengua como un fenómeno multicéntrico e internacional y que, en consonancia, proyecta un modelo de gestión multilateral (OLIVEIRA, 2015, p. 32). En relación con la orientación adoptada por la institución en los últimos años, Lagares (2016, p. 292) observa que los esfuerzos se han centrado más en la planificación del estatus internacional del portugués que en la del corpus y que ha sido muy marcada "la intervención política de los estados que integran la CPLP y de sus instituciones en las decisiones que afectan a la lengua", como ocurrió con el AOLP. Por su parte, Carvalho (2012) destaca que el IILP ha buscado debatir las cuestiones lingüísticas dentro del área más allá del tradicional binomio Brasil-Portugal, favoreciendo la inclusión del resto de los países lusófonos.

Finalmente, para Faraco (2016, p. 357) es importante explotar el potencial del IILP como órgano de coordinación y gestión multilateral, esto es, no de carácter ejecutivo sino como espacio de planificación de acciones y de articulación con las instituciones competentes para realizar cada una de ellas. Entiende que el proyecto del Vocabulario Ortográfico Común de la Lengua Portuguesa, cuya elaboración fue proyectada en las bases del AOLP, es una buena iniciativa en tal sentido. ${ }^{6}$ De acuerdo con Correia y Ferreira (2012), la propuesta va a significar una oportunidad

6. Sobre el proceso de elaboración del Vocabulario, ver Almeida, Ferreira, Correia y Oliveira (2013). Desde mayo de 2016, la base lexical está disponible online (http://voc.cplp.org/index. php?action=apresent). Acceso: 15 jul. 2018. 
para estabilizar la lengua portuguesa y consolidarla como lengua internacional y pluricéntrica.

\subsection{Política lingüística exterior e integración regional}

Como sabemos, la CPLP como organismo que materializa la política de área lusófona es un espacio supranacional conformado por un conjunto de países reunidos, en principio, por un criterio político-lingüístico: tener el portugués como lengua oficial. A la vez, estos Estados integran -y algunos de ellos comparten- distintos bloques regionales. Lo anterior nos lleva a interrogarnos, en esta parte del trabajo, sobre la relación entre los miembros del área idiomática lusófona, especialmente en lo que concierne a Portugal y a Brasil, y las integraciones regionales de las que participan.

En el caso de Portugal, la disyuntiva parece estar entre enfocarse exclusivamente en la Unión Europea o -sin dejar de interesarse por este objetivoacercarse a África y a América Latina. Respecto de la situación de Brasil, su política lingüística de proyección internacional ha convivido con otras en ámbitos regionales, centradas en la integración sudamericana en el contexto del Mercosur, y con una geopolítica multilateral sur-sur o con énfasis en el eje de países emergentes conocido como BRICS (Brasil, Rusia, India, China y Sudáfrica) (FARACO, 2016).

En primer lugar, Castellanos Brieva (2013) examina la importancia de dos entidades supranacionales que integra Brasil, la CPLP y el Mercosur, para el desarrollo del portugués a nivel mundial: en el primer caso, a partir de la promoción de una imagen de lengua internacional; el segundo, orientado al fomento del bilingüismo español/ portugués en la región. En esta dirección, Arnoux (2014) exhibe el funcionamiento de las ideologías lingüísticas a partir del anclaje en diferentes representaciones sociolingüísticas, como puede ser la construcción del portugués como lengua asociada a un territorio nacional, desde el sector estatal brasileño que se ocupa del Mercosur, y la búsqueda de porosidad de los límites, de desterritorialización, desde una perspectiva que se interesa por la expansión global del portugués.

Continuando con el papel del Mercosur, en especial en lo que atañe a la construcción de una región bilingüe, Carvalho (2012) y Carvalho y Schlatter (2011) destacan el proyecto de la Escuela Intercultural Bilingüe de Frontera y la Universidad Federal de la Integración Latinoamericana (UNILA). El primero, que tiene por objetivo promover el intercambio y la integración entre profesores y estudiantes de los miembros del bloque, es resultado de un acuerdo bilateral entre los gobiernos brasileño y argentino firmado en 2005 y luego ampliado a otros territorios de la región. En este marco también se inscribe el Observatorio de Educación en la Frontera cuyo propósito es la educación lingüística en los 
contextos fronterizos de Brasil y los territorios hispanos, enfocando la diversidad lingüística en esos espacios y su relación con el ámbito educativo y el entorno de las comunidades involucradas. En el caso de la UNILA, guiada por una política de educación bilingüe en español y en portugués, tiene como propósito promover la cooperación y el intercambio de conocimiento y cultura entre los países de América Latina. De acuerdo con Carvalho (2012, p. 481), este tipo de acciones favorece la valorización de los entornos multilingües y promueve una nueva visión de la relación entre lenguas, contribuyendo a la construcción de una conciencia multilingüe, que destaca positivamente la convivencia con otras lenguas.

Por su parte, Diniz (2012) considera que, aunque el eje principal de la política externa brasileña ha sido, históricamente, América del Sur, a partir del gobierno de Luiz Inácio Lula da Silva esta región adquirió una centralidad excepcional, en el marco de un proyecto político de integración. En los años siguientes hubo una aproximación a América Central y el Caribe. En relación con los discursos que circulan en Brasil en torno a las gestiones de promoción exterior del portugués, Diniz (2016) muestra que estas suelen ser significadas como neocolonizadoras y neoimperialistas. Interrogando el espacio del Mercosur en particular, el autor señala la necesidad de lograr una mejor comprensión -y una visualización- de los imaginarios asociados al portugués y a las otras lenguas que conviven en esa región, anclados en memorias que se (re)configuran socio-históricamente en función de las distintas coyunturas (DINIZ, 2016, p. 110-111).

Continuando con el planteo de Diniz (2012, p. 445), históricamente, los proyectos del Ministerio de Asuntos Exteriores de Brasil favorecieron la propagación de la lengua portuguesa en América Latina a través de los centros e institutos culturales, mientras que los emprendimientos en Europa se vincularon con la universidad pública a través de los lectorados. Sin embargo, el panorama ha ido cambiando en la primera década del siglo XXI con la expansión de la red de lectorados hacia América Latina, sobre todo del Cono Sur, lo que muestra la importancia que adquiere la región en la política externa de Brasil (DINIZ, 2012).

En palabras de Faraco (2012, p. 43), para este país, desde el punto de vista diplomático, la CPLP "representa basicamente uma opção estratégica para articular, com base na língua comum, uma cooperação Sul-Sul (Brasil - países africanos de língua oficial portuguesa - Timor) com a vantagem de ter um vértice na União Europeia (Portugal)". De acuerdo con Lopes (2013, p. 28-29), el portugués ha comenzado a ocupar un nuevo lugar en el mercado de lenguas vinculado, entre otras cuestiones, al papel de Brasil en el bloque regional BRICS y al interés que ha despertado internacionalmente "la exploración de riqueza" de algunos países 
africanos de habla oficial portuguesa. Una de las iniciativas por parte del Estado brasileño orientada a la integración cultural y de cooperación internacional Sur-Sur fue la creación de la Universidad de la Integración Internacional de la Lusofonía Afro-Brasileña (UNILAB) en el año 2010 (GOMES y VIEIRA, 2013). Por su parte, Diniz y Bizón (2015) plantean que el fortalecimiento, a principios del siglo XXI, de la presencia de los Centros Culturales Brasileños en el continente africano y del número de participantes del Programa de Estudiantes-Convenio de Graduación provenientes de los PALOP da cuenta de que el establecimiento de alianzas con África fue una de las líneas estratégicas de la política exterior del gobierno de Lula da Silva.

En cambio, para Fiorin (2013) la pertenencia de Portugal y Brasil a dos integraciones diferentes - la Unión Europea y el Mercosur, respectivamente- aleja a la CPLP de la transformación en un espacio económico de libre circulación de bienes. De modo que solo puede ser un espacio de unidad política, lingüística y cultural, para lo cual es necesario forjar una identidad común, que no debe ser el resultado de la asimilación de una identidad dentro de otra, es decir, no debe dejar de lado ninguna identidad.

Respecto de la situación de Portugal, según Faraco (2012) los debates están atravesados por un dilema de naturaleza geopolítica. Para algunos el centro de interés debe estar en la adecuada integración del país en la Unión Europea; desde esta perspectiva, el Estado debería mantener relaciones económicas y culturales con los otros territorios donde el portugués es idioma oficial, pero sin unirse formalmente a otro bloque internacional que pueda traer dificultades al entorno de la Unión Europea (FARACO, 2012, p. 44). Por otro lado, están los que, teniendo en cuenta que Portugal tiene un peso político y económico relativamente pequeño en este bloque regional, sostienen la importancia de un compromiso fuerte del país en la CPLP, de modo que esto pueda darle impulso político y económico dentro de la Unión Europea, en la cual pasaría a ser visto como eslabón facilitador de las relaciones con el Mercosur, África y Asia (FARACO, 2012, p. 45). Desde la perspectiva de Silva (2010b), el despliegue de la política lingüística exterior de Portugal ha sido sobre todo a través del Instituto Camões y se ha centrado más en los continentes europeo, africano y asiático, a diferencia de Brasil que puso el foco en América Latina.

\section{LA LUSOFONÍA: PERSPECTIVAS, LÍMITES Y TENSIONES}

Siguiendo el planteo de Lagares (2013, p. 388), las "fonías" -entre ellas, la hispanofonía, la francofonía o la lusofonía- son espacios de gestión internacional 
de lenguas que constituyen productos de procesos históricos de expansión política y/o religiosa sobre determinados territorios. Se trata de espacios atravesados, de un modo u otro, por pugnas o tensiones que han derivado de dichos procesos. Como consecuencia de las relaciones de fuerza al interior del área, se generan "posiciones jerárquicamente diferenciadas entre países centrales, secundarios y periféricos a pesar de las declaraciones respecto de la gestión democrática de la lengua compartida" (ARNOUX, 2018, p. 34) que sostienen las políticas de áreas idiomáticas. Desde el punto de vista lingüístico, se produce una situación de hegemonía política y social de una lengua, lo que conlleva la minorización lingüística de las otras lenguas del territorio y de sus hablantes. A continuación, revisaremos algunas concepciones y perspectivas sobre el término lusofonía presentes en un conjunto de estudios y luego haremos un recorrido por las evaluaciones que realizan los autores sobre los proyectos y las políticas de gestión internacional del portugués o sobre el modo en que estos son recibidos, donde opera, de diversas maneras, la interpretación en clave neocolonizadora, o su rechazo.

\subsection{Enfoques sobre el término lusofonía}

Una de las primeras referencias que surge al reflexionar sobre la noción de lusofonía es la del libro clásico del profesor e investigador portugués Fernando Cristóvão (2008, p. 109), quien allí la define como "o conjunto dos oito países e de algumas regiões em que a língua portuguesa é usada como materna ou oficial e que, por opção livre, querem colaborar entre si e formarem um grupo para as iniciativas de qualquer tipo em que estiverem de acordo". Dentro de esta perspectiva que privilegia la concepción de la lusofonía como imaginario identitario, incluimos los trabajos de Martins (2014, p. 25), quien considera que se trata de un "movimento multicultural de povos que falam a mesma língua, o portugués" y agrega que la lusofonía, "ao invés da homogeneização empobrecedora e de sentido único, estabelecida pela globalização cosmopolita, tem a virtude do heterogéneo". En consecuencia, no hay un imaginario único de comunidad lusófona sino múltiples (MARTINS, 2015). El autor también señala ciertos equívocos que atraviesan el término lusofonía y que es preciso deconstruir, entre ellos, el de una centralidad portuguesa (MARTINS, 2006, 2014) al que nos referiremos en el apartado siguiente.

En esta dirección, hay quienes interrogan las fronteras de la lusofonía y de la CPLP en tanto institución que la representa y proponen que los límites de aquella deberían ir más allá del conjunto de países que integran este organismo a partir de la incorporación de otros grupos, comunidades o regiones que tienen lazos con el portugués y con la cultura lusófona. De acuerdo con Pinto (2011), por ejemplo, 
para que la CPLP constituya una comunidad lusófona debe poder albergar no solo países, sino también comunidades y regiones, es decir, pueblos que tienen filiaciones con aquella cultura, como Galicia ${ }^{7}$ o Macao. Para Cristóvão (2014), dado que los límites de la lusofonía van más allá de los territorios nacionales con portugués como lengua oficial, e incluyen la emigración portuguesa en el mundo y los extranjeros que lo utilizan como segunda lengua o lengua extranjera, entre otros, el IILP debería constituirse en una institución independiente que, siguiendo los lineamientos de la CPLP respecto de la cultura y la lengua, pudiera organizarse como una suerte de Gran Academia ("uma Academia das Academias lusófonas") a la que pertenecerían las academias nacionales u otras instituciones afines en articulación con universidades, centros de investigación de lingüística, de literatura, de historia, entre otros.

Otro conjunto de estudios concibe la lusofonía como una construcción que responde a ciertos intereses políticos vinculados con el área idiomática del portugués. Dentro de este enfoque se destaca el libro de Carlos Alberto Faraco (2016) donde se explica el proceso histórico e ideológico de construcción de la noción de lusofonía, que toma como base la lengua común extendida por el antiguo imperio portugués para el despliegue de un proyecto político de carácter poscolonial. Tanto Faraco (2012, 2016) como Bagno (2009) mantienen una postura crítica frente a la lusofonía: el primero plantea que, en la actualidad, esta no pasa de ser una quimera (FARACO, 2016, p. 327); para Bagno (2009) está más cerca de ser una ilusión que una realidad -para dar cuenta de ello usa la expresión ilusofonia- ya que se basa en "entidades inócuas" y en "um discurso de samba-exaltação sem consequências concretas".

Por su parte, Carneiro (2013) considera que la centralidad dada a la lengua portuguesa en el uso de la palabra lusofonía tiende a invisibilizar conflictos derivados del proceso histórico de dominación colonial y la condición multilingüe de los territorios comprendidos en la CPLP. En tal sentido, Fabrício (2013) sostiene que la noción tiene que ser resignificada tomando distancia de la visión tradicional colonialista en la que está inmersa y de concepciones estereotipadas y utópicas de unión cultural de los pueblos de habla portuguesa. En una orientación similar, para Orlandi (2012, p. 12) el término trae la memoria de la colonización y remite a una homogeneidad que no existe, por lo tanto, es preciso redefinir este espacio lingüístico como "conjunto de línguas que, no presente, é um campo dinâmico e

7. En el año 2014 el Consejo de la Cultura Gallega fue admitido como observador consultivo de la CPLP. La nueva función hizo que Galicia pudiera estar presente en las reuniones temáticas de la entidad, establecer vínculos con esta red de estados y servir de enlace permanente entre la entidad y la cultura y lengua gallegas. 
diversificado, multilingue, na sua coexistência com uma língua européia que faz parte desta história", para dar cuenta de nuevos modos de reorganizar las relaciones y el funcionamiento de las lenguas en el presente. Por último, en la misma línea, Severo $(2016$, p. 1331) señala que la lusofonía envuelve relaciones de poder, por lo tanto, asimetrías, y "mobiliza uma série de elementos simbólicos interligados, se apropriando, fundamentalmente, da 'língua portuguesa' como recurso político poderoso que reforçou e reforça modos de diferenciação e de classificação dos sujeitos". Estas últimas posiciones exhiben la necesidad de repensar la lusofonía apartándose de miradas colonizadoras y homogeneizadoras que, como veremos en los dos apartados que siguen, atraviesan de modo variado distintas acciones y proyectos de la política internacional del portugués.

\subsection{Tensiones en torno al eje (ex)dominador/(ex)dominado}

Los análisis sobre los debates y las implicancias del AOLP trazan lecturas que activan, de una u otra forma, la memoria de la colonización. A lo largo del siglo $\mathrm{XX}$, el proceso histórico en materia ortográfica estuvo guiado por una voluntad de ruptura del pasado colonial por parte de Brasil que lo llevó a diferenciarse a partir de la acentuación de lo nacional. Pero con el avance de los procesos de globalización, que impulsan importantes desafíos para las lenguas mayores, las cuales deben competir en el espacio global y adquieren relevancia para los nuevos mercados transnacionales, este país apostó a la unificación ortográfica. Así, a diferencia de las reformas ortográficas desarrolladas en el ámbito lusófono hasta mediados del siglo pasado, cuyas iniciativas fueron impulsadas desde los ámbitos nacionales y sujetas a los procesos legislativos de Brasil y de Portugal (Lagares, 2018), el AOLP supuso un trabajo conjunto, aunque no por ello armónico y equitativo, entre ambos países, así como apoyo y aceptación estatal multilateral.

Recordemos que el objetivo del acuerdo, firmado en 1990, fue unificar las dos ortografías oficiales existentes del portugués, la europea y la brasileña, creando una base común que favoreciera una mayor circulación de materiales producidos en esta lengua. El AOLP, cuyos pilares fueron elaborados por la Academia de Ciencias de Lisboa y por la Academia Brasileña de Letras, contó con la firma de representantes oficiales de Angola, Brasil, Cabo Verde, Guinea-Bissau, Mozambique, Portugal y Santo Tomé y Príncipe. ${ }^{8}$ Durante el largo proceso de elaboración del acuerdo, que duró muchos años debido a que su entrada en vigencia recién comenzó al finalizar

8. Más adelante, en 2004, Timor Oriental adhirió al acuerdo. Dos años antes había ingresado a la CPLP, una vez alcanzada su independencia. 
la primera década del siglo XXI y según los tiempos de cada Estado, se desataron encendidos debates y expresiones de rechazo -mayormente en Portugal- que incluso perduran en la actualidad. ${ }^{9}$

En términos de Fiorin $(2009,2010,2013)$, el AOLP constituye un instrumento político de construcción de una identidad común basada en la aceptación de la diversidad. En el plano lingüístico, para lograr este propósito es necesario que la lengua constituya "um espaço em que todas as variedades linguísticas sejam, respeitosamente, tratadas em pé de igualdade", sin autoridad paterna lusitana (FIORIN, 2010, p. 66). Continuando con el planteo del autor, las polémicas que desató el tratamiento del acuerdo - desde Brasil la tendencia fue la indiferencia hacia la exmetrópoli, mientras que en Portugal los argumentos de rechazo apelaron a la pureza de la lengua y la pretensión de hegemonía de la legitimidad lingüísticaexpusieron una rivalidad existente entre los dos países y la incapacidad para construir una identidad lusófona común. En una dirección similar, Silva (2011) considera que el acuerdo removió "heridas mal cicatrizadas" entre estos países, las cuales remiten a los vínculos entre excolonizador y excolonizado. En palabras de Lagares (2018, p. 193), en Portugal el acuerdo fue interpretado como "uma espécie de abrasileiramento da língua e, portanto, como uma operação de imperialismo cultural empreendido pela antiga colônia", invirtiendo el tradicional vínculo de dominación portuguesa del (ex)colonizador hacia el (ex)colonizado.

Los debates y las repercusiones en torno a la adopción de una ortografía común para toda el área lusófona así como la creación y el desarrollo de la CPLP también han reavivado la cuestión, de larga trayectoria histórica, acerca de la conveniencia de unificación de la norma idiomática o, por el contrario, de conservación de normas divergentes. En este sentido, algunos investigadores brasileños adoptan un posicionamiento bien marcado en materia de soberanía idiomática al argumentar, de diferente modo, que el portugués de su país y el de Portugal son dos lenguas diferentes (ORLANDI, 2009; BAGNO, 2013, 2018)..$^{10}$ De acuerdo con Orlandi (2009, p. 172), en el traspaso de la lengua portuguesa para Brasil se produjo un proceso de historización que dio como resultado una descolonización lingüística, donde el lugar de memoria por la cual se significa la lengua deja de ser Portugal

9. Un ejemplo de esto es la "Iniciativa legislativa de Cidadãos contra o Acordo Ortográfico" que, impulsada en el año 2008 a través de internet (https://ilcao.com/, acceso: 15 jul. 2018), busca juntar 20.000 firmas para llevar a tratamiento parlamentar un proyecto de Ley que revoque la entrada en vigor del AOLP en Portugal.

10. Para dar cuenta de esta especificidad, Orlandi (2009) propone el sintagma lengua brasileña. En el mismo sentido pueden interpretarse los títulos de las siguientes obras: Bagno (2012) Gramática pedagógica do português brasileiro y Bagno y Carvalho (2015) Gramática brasileña para bablantes de español. 
y pasa a ser la memoria local de Brasil. Bagno (2018), por su parte, a partir de la consideración del gallego -y no el latín-como origen del portugués y de la distinción de rasgos fonético-fonológicos y morfosintácticos específicos, sostiene la tesis de que el portugués brasileño y el portugués europeo son dos sistemas lingüísticos distintos que deben ser objetos de políticas lingüísticas diferenciadas.

En cambio, Oliveira (2013a, 2013b, p. 69), situado en otra perspectiva, señala que la normatización divergente -el portugués europeo y el brasileño- es cada vez más disfuncional ya que las lenguas tienden a ser más centrales en el mundo actual. En este rumbo, para el autor el AOLP es un primer paso hacia un nuevo modo de gestión de la lengua -internacional, convergente, multilateral y comunitario, esto es, congruente con el marco de la CPLP (OLIVEIRA, 2013b) - a partir de la construcción de una norma común, la cual, a su entender, no resultaría de la imposición de una forma central o única del idioma sino que derivaría de instancias comunes de gestión y deliberación. Para ello, en términos de Lagares (2016, p. 293), los Estados que integran la CPLP tendrían que ceder "a esa institución la autoridad y los medios necesarios para emprender una normativización convergente de esas características".

En relación con el papel de la CPLP, entre los aspectos de este organismo más cuestionados se destaca la asociación de la entidad y de la idea de lusofonía que vehiculiza con los intereses de Portugal (ORLANDI, 2012; CARNEIRO, 2013; FABRÍCIO, 2013; FARACO, 2016). Según Faraco (2016, p. 314), la voluntad de reunir políticamente a los Estados con lengua oficial portuguesa provino principalmente de un interés político-diplomático del país europeo. En cambio, Castro (2010, p. 67), desde otra posición enunciativa, plantea que la lusofonía y las acciones vinculadas a ella, como el AOLP o las que emanan de instituciones como la CPLP, no tienen que ver con una gestión poscolonial y tampoco parecen ser resultado de impulsos portugueses. Del mismo modo, Cristóvão expresa lo siguiente: "Não é, pois, a Lusofonia uma forma de neocolonialismo cultural, é uma pátria comum onde as diferenças se completam numa unidade de iniciativas em face da pressão cada vez maior da globalização, impedindo assim os efeitos descaracterizadores desta, preservando e valorizando o que cada país sozinho não podia realizar" (2008, p. 109).

En los discursos de difusión del portugués como lengua internacional que produce la CPLP también se identifica una mirada colonialista. A partir del análisis de documentos vinculados a la constitución de este organismo, Signorini (2013) compara dos ideologías lingüísticas: la globalista portuguesa, que se asienta en una retórica colonial, basada en el monolingüismo; y la globalista brasileña que, tomando 
distancia de la primera, tiende al fomento de relaciones bilaterales o multilaterales con diferentes países, pero sin otorgar un lugar especial a los de la CPLP. Frente a este panorama, la autora vislumbra la posibilidad de orientarse hacia un modelo transnacional, transcultural y transidiomático que se distanciaría de los anteriores, en el marco del poscolonialismo y de la descolonización.

Respecto del papel específico de Brasil en la política internacional de promoción de la lengua portuguesa, Diniz (2016, p. 101) observa que en los discursos de difusión resuena comúnmente la construcción de este país como neocolonizador y neoimperialista, especialmente en el ámbito de los PALOP y de América, que han sufrido políticas coloniales e imperialistas. Para incidir en esta situación, el autor propone lograr, en el espacio del Mercosur en particular, una mejor comprensión y una visualización de los imaginarios asociados al portugués y a las otras lenguas que conviven en esa región, reflejando las tensiones entre ellas, con el propósito de superar "os objetivos puramente mercadológicos desse bloco" y trabajar en favor de la construcción del portugués como lengua de integración (DINIZ, 2016, p. 111).

En lo que concierne al Instituto Camões ${ }^{11}$, como principal organismo que ha canalizado las iniciativas de expansión internacional de la lengua portuguesa por parte de Portugal, sus acciones también han sido vinculadas a intenciones neocolonialistas. En tal sentido, Cristóvão planteaba en 2010 que la entidad debía reconvertirse y reorientar sus acciones de modo de tomar distancia de cualquier interpretación de este tipo. Para ello, el autor propone el diseño de proyectos compartidos, por ejemplo, en el traslado de profesores y lectores o en la edición conjunta de obras, no sólo sobre el portugués, sino también sobre las otras lenguas de los países que antes eran considerados periféricos (CRISTÓVÃO, 2010, p. 52).

\subsection{Tendencias homogeneizantes y valoración del multilingüismo}

En el plano lingüístico, la tensión entre dominador y dominado repercute en otra tensión que incluye la antinomia homogeneidad/heterogeneidad. En líneas generales, los estudios sobre política internacional del portugués identifican, por

11.Durante el año 2012, en el contexto de racionalización de los recursos económicos que trajo aparejada la crisis mundial, se produjo la fusión entre el Instituto Camões y el Instituto Portugués de Apoyo al Desenvolvimiento dedicado a políticas de cooperación, lo que dio como resultado un organismo bicéfalo: Instituto Camões-Instituto de la Cooperación y de la Lengua, I. P., dependiente del Ministerio de Negocios Extranjeros. De acuerdo con Alves y Araújo (2015, p. 62), esta acción no fue bien recibida desde ciertos sectores políticos, diplomáticos y académicos en la medida en que dejaba a la cultura nacional portuguesa en una posición subalterna respecto de otros países lusohablantes. 
un lado, tendencias homogeneizantes en determinadas acciones y proyectos que buscan imponer "una" realidad dominante y, por otro, expresan la necesidad de abandonar esta orientación y adoptar una perspectiva atenta a la heterogeneidad, que pueda contemplar tanto las diferencias dentro del portugués como la realidad multilingüe del área lusófona, especialmente en los países africanos donde la lengua portuguesa es oficial pero no mayoritaria. Siguiendo este recorrido, los estudios coinciden -si bien desde variados puntos de partida y perspectivas teóricasen que la lusofonía solo puede ser viable si apunta a constituirse como espacio heterogéneo, multicultural y se aleja de representaciones estereotipadas (BRITO, 2015, p. 298; MARTINS, 2006; FIORIN, 2009; FABRÍCIO, 2013; CARNEIRO, 2013; ORLANDI, 2012).

En primer lugar, Silva (2013) analiza algunos documentos de intenciones difundidos en los foros realizados en el marco de la CPLP y observa una propensión hacia la producción de efectos de homogeneidad y de ocultamiento de la heterogeneidad, silenciando las diferencias en torno de los sentidos de lengua portuguesa. De este modo, el autor constata un proceso de homogeneización de la lengua portuguesa orientado a atender al mercado global, donde el portugués brasileño gana espacio como base de esa lengua. Sin embargo, esta tendencia coexiste con otra, producto de los procesos de gramatización de las diferentes lenguas portuguesas nacionales, que valora la heterogeneidad (SILVA y SANT'ANNA, 2012, p. 132). En otro trabajo, Silva (2012) estudia proyectos de cooperación técnica en educación ofrecidos por Brasil a países en desarrollo de la CPLP, como Timor Oriental, y concluye que, a pesar de que se evidencia un discurso de solidaridad, la cooperación brasileña construye un sentido de "transferencia" y, por lo tanto, una verticalidad que no da lugar a la construcción conjunta entre las partes involucradas. En esta falta de espacio para la intervención por parte del país que es "asistido" también se manifiesta una tendencia homogeneizante (SILVA, 2012, p. 145), en línea con los vínculos asimétricos que comentamos en el apartado anterior.

En relación con los PALOP, para Severo (2015, p. 101), el hecho de que en los territorios africanos el idioma portugués sea, aunque oficial, cuantitativamente minoritario, sería una señal de los límites de la lusofonía como ideal homogeneizador. En tal dirección, uno de los principales desafíos que destacan los estudios es garantizar que las políticas en el marco de la lusofonía no silencien las otras lenguas nacionales sino que respeten la condición multilingüe de esos territorios (COUTO, 2009; BRITO, 2010; HANNA, BRITO y BASTOS, 2010; REIS, 2015). Sobre la situación particular de Mozambique, Couto (2009) plantea que para que el proyecto de la lusofonía funcione en este país, no puede ser considerado como algo vinculado 
con los intereses de grupos portugueses sino que debe orientarse a la defensa de las otras lenguas y culturas que conviven con el portugués en Mozambique.

Por su parte, Diniz y Bizón (2015) analizan dos políticas de promoción internacional del portugués en los países africanos donde es idioma oficial e identifican la construcción de un imaginario de hermandad entre Brasil y África que silencia los intereses geopolíticos y económicos de la política externa de aquel. Respecto del Programa de Estudiantes-Convenio de Graduación, los autores encuentran políticamente equivocada la decisión de exigir el Celpe-Bras para los candidatos procedentes de la CPLP porque, por un lado, dado que el examen no fue concebido para la evaluación de lengua primera, su aplicación para públicos diferentes de aquellos para los cuales fue diseñado trae dificultades; por otro lado, la disposición en cuestión significa contribuir a un proceso de capitalización lingüística (ZOPPI-FONTANA, 2009, 2010) del portugués brasileño, de modo que lo coloca en el centro del "habla portuguesa" $y$, en consecuencia, descapitaliza otras variedades portuguesas de África. Este posicionamiento, al apagar justamente la posibilidad de que el convenio funcione sobre bases de una cooperación multilateral, refuerza la imagen de Brasil como un país neocolonizador (DINIZ y BIZÓN, 2015).

Finalmente, Reis (2015, p. 17) observa que en el uso de la expresión "espacios" de la lengua portuguesa, en plural, se producen tensiones inevitables que remiten a la "dialética entre centro e periferia e entre conservação normativa e inovação transgressora". Desde una perspectiva optimista, el uso del plural lleva a pensar en diferencias que enriquecen el idioma pero esa dinámica de pluralidad y diversificación de la lengua portuguesa también conlleva ciertos desafíos, entre ellos, la cuestión-que, como vimos, han abordado otros autores de un modo más o menos explícito-respecto de quién rige y con qué autoridad -en términos de instituciones, según el autor, no de territorios nacionales- el proceso de internacionalización de la lengua portuguesa (REIS, 2015, p. 17).

\section{REFLEXIONES FINALES}

En este artículo hemos revisado investigaciones realizadas en los últimos años sobre la política lingüística internacional del portugués. Situados en diferentes lugares de enunciación y, en general, desde variadas perspectivas teóricas, los trabajos comparten un interés común por evaluar e interrogar las acciones en torno al desarrollo transnacional del portugués, emprendidas desde instancias de intervención nacional o desde el área lusófona. 
En líneas generales, los autores aquí reunidos -con escasas excepcionesdestacan la vigencia de tensiones derivadas del proceso histórico de dominación colonial que inciden de un modo u otro en las actuales políticas lingüísticas. De esta forma, se detectan tendencias neocolonizadoras - no solo por parte de la antigua exmetrópoli sino también de Brasil- en proyectos y acciones que, apoyadas en una representación de la lusofonía como entidad homogénea, monolingüe, más cercana al imaginario de Estado-nación, actúan en detrimento de una gestión multilateral del portugués más adecuada a la realidad. Aquellas tensiones son, a nuestro entender, constitutivas del espacio de la lusofonía -atravesado a su vez por distintas disputas ideológicas- $y$, por lo tanto, no pueden ser desestimadas. En este sentido, el principal desafío parece dirigirse a repensar la lusofonía así como los proyectos e instituciones vinculadas a ella tomando distancia de concepciones homogeneizadoras y reelaborando los ecos colonialistas a partir de la construcción de relaciones multilaterales dinámicas entre agentes/instituciones/países y del despliegue de imaginarios identitarios que visibilicen la realidad heterogénea y multilingüe de los territorios lusófonos.

Los trabajos coinciden, a grandes rasgos, en la falta de coordinación entre acciones nacionales y entre estas e iniciativas multilaterales y abogan por el diseño de políticas lingüísticas congruentes con los diversos contextos sociolingüísticos del área lusófona y por la construcción de una gestión multilateral del portugués más equilibrada. Algunos autores, sin embargo, se apartan en este último punto y muestran una inclinación hacia algún centro rector. De modo más o menos explícito, los lugares de enunciación desde donde se producen los estudios inciden en los análisis que los investigadores realizan de las políticas lingüísticas así como en las propuestas glotopolíticas que formulan.

Para finalizar, advertimos que el panorama presentado en este artículo ha sufrido transformaciones en los últimos años, vinculadas principalmente con los cambios producidos en el contexto de la crisis institucional, política y económica de Brasil, por lo que sería deseable la incorporación de nuevas investigaciones que contribuyan a la actualización y discusión de políticas internacionales del portugués.

\section{REFERENCIAS}

ALMEIDA, G. B.; FERREIRA, J. P.; CORREIA, M.; OLIVEIRA, G. M. (2013). Vocabulário Ortográfico Comum (VOC): constituição de uma base lexical para a língua portuguesa. Revista de Estudos Linguísticos. v. 42, nº 1, pp. 204-215. 
ALVES, L. A.; ARAúJO, F. M. (2015). Camões, Instituto da Cooperaçao e da Língua (Portugal). Revista Internacional de Lingüística Iberoamericana, v. XIII, $\mathrm{n}^{\mathrm{O}} 1$ (25), pp. 53-66.

ARNOUX, E. N. de (2000). La glotopolítica: transformaciones en un campo disciplinario. Revista Lenguajes: teorías y prácticas. $n^{\circ} 1$, pp. 3-27.

ARNOUX, E. N. de (2011). Desde Iguazú: mirada glotopolítica sobre la integración regional. En: Fanjul, A.; Castela, G. da Silva (orgs), Linguas, políticas e ensino na integração regional. Cascavel: ASSOESTE, pp. 38-64.

ARNOUX, E. N. de (2014). Glotopolítica: delimitación del campo y discusiones actuales con particular referencia a Sudamérica. En: Zajícová, L.; Zámec, R. (eds.), Lengua y política en América Latina: perspectivas actuales. Olomouc: Univerzita Palackého v Olomouci, pp. 19-43.

ARNOUX, E. N. de (2018). Cómo el proceso de integración regional interroga a la Glotopolítica. En: Sousa, E. S. C. Tavares de; Roca, M. P.; Ponte, A. (orgs.), Temas de política linguística no processo de integração regional. Campinas: Pontes, pp. 21-45.

ARNOUX, E. N. de; DEL VALLE, J. (2010). Las representaciones ideológicas del lenguaje. Discurso glotopolítico y panhispanismo. Spanish in Context. v. 7, n ${ }^{\circ} 1$, pp. 1-24.

BAGNO, M. (2009). Lusofonia ou ilusofonia? Caros amigos. A primeira á esquerda.

BAGNO, M. (2013). Viva o acordo ortográfico. Aplauso, pp. 42-43.

BAGNO, M. (2018). Duas línguas, quantas políticas? En: Sousa, E. S. C. Tavares de; Roca, M. P.; Ponte, A. (orgs.), Temas de política linguística no processo de integração regional. Campinas: Pontes, pp. 47-65.

BAGNO, M.; CARVALHO, O. L. S. (2015). O potencial do português brasileiro como língua internacional. Interdisciplinar: Revista de Estudos em Língua e Literatura, v. 22, pp. 11 26.

BRITO, R. P. (2010). Moçambique e Timor Leste: realidade multílingue e oficialidade lusófona. En: Marçalo, M. J. et al. (eds.), Língua portuguesa: ultrapassar fronteiras, juntar culturas. Évora: Universidade de Évora, pp. 71-92.

BRITO, R. P. (2015). "À mistura estão as pessoas": Lusofonia, política linguística e internacionalização. En: Martins, M. L. (coord.), Lusofonía e interculturalidade. Promessa e travessia. CECS: Universidad de Minho, pp. 295-312. 
BRITO, R. P.; BASTOS, N. (2011). Percepções da Lusofonia em portais governamentais. En: Martins, M. L.; Cabecinhas, R.; Macedo, L. (eds.), Anuário Internacional de comunicação lusófona. Lusofonía e Cultura-Mundo. Braga: Grácio Editor, pp. 105-119.

CALVET, L.-J.; CALVET, A. (2013). Les confettis de Babel. Diversité linguistique et politique des langues. Montreal: Écriture.

CARNEIRO, A. S. R. (2013). Conflitos em torno da (des)construção da(s) língua(s) legítima(s): a situação da língua portuguesa no contexto multilíngue de TimorLeste. En: Lopes, L. P. da Moita (org.), O português no século XXI. Cenário geopolítico e sociolinguístico. San Pablo: Parábola, pp. 192-217.

CARVALHO, S. da Costa (2012). Políticas de promoção internacional da língua portuguesa: ações na América Latina. Trabalhos em Linguística Aplicada, v. 51, n 2, pp. 459-484.

CARVALHO, S. da Costa; SCHLATTER, M. (2011). Ações de difusão internacional da língua portuguesa. Cadernos do IL. n. ${ }^{\circ}$ 42, pp. 260-284.

CASTELLANOS BRIEVA, J. P. (2013). Política lingüística del portugués en Brasil: del colonialismo al siglo XXI. Cuadernos de Lingüística Hispánica. n² 21, pp. 27-40.

CASTRO, I. (2008). Galiza no espaço cultural e simbólico da Lusofonia. Grial. v. 46, nº 180, pp. 80-89.

CASTRO, I. (2010). As Políticas Linguísticas do Português. XXV Encontro da Associação Portuguesa de Linguística. Porto: APL, pp. 65-71.

CORREIA, M.; FERREIRA, J. P. (2013). Dicionários e vocabulários ortográficos na constituição da norma. En: Lopes, L. P. da Moita (org.), O português no século XXI. Cenário geopolítico e sociolinguístico. San Pablo: Parábola, pp. 297-318.

COUTO, M. (2009). Ese Obama fosse africano? Eoutras interinvenções. Lisboa: Editorial Caminho.

CRISTÓVÃO, F. (2008). Da lusitanidade à lusofonia. Coimbra: Almedina.

CRISTÓVÃO, F. (2010). A lusofonia desta década no meio de algumas contradições. Letras Com vida. Literatura, cultura e arte. $\mathrm{n}^{\mathrm{O}} 2$, pp. 47-54.

CRISTÓVÃO, F. (2014). Uma Academia das Academias lusófonas para a língua portuguesa. Ciberdúvidas da língua portuguesa. Disponible en: https://ciberduvidas.iscte-iul.pt/ artigos/rubricas/lusofonias/uma-academia-das-academias-lusofonas-para-a-linguaportuguesa/3084. Acceso: 15 jun. 2018. 
DEL VALLE, J. (2017). La perspectiva glotopolítica y la normatividad. AGlo. Anuario de Glotopolítica, nº 1, pp. 17-39.

DINIZ, L. R. A. (2012). Políticas linguísticas do Estado brasileiro para a divulgação do português em países de língua oficial espanhola. Trabalbos em Linguística Aplicada, v. 51, $\mathrm{n}^{\mathrm{O}} 2$, pp. 435-458

DINIZ, L. R. A. (2016). A promoção do português no espaço de enunciação do Mercosul: alguns equívocos históricos estruturantes. En: Arnoux, E. N.; Lauria, D. (comps.), Lenguas y discursos en la construcción de la ciudadanía sudamericana. Gonnet: UNIPE, pp. $99-$ 113.

DINIZ, L. R. A.; COSSI BIZÓN, A. C. (2015). Discursos sobre a relação Brasil/Africa "lusófona" em políticas linguísticas e de cooperação educacional. Línguas e instrumentos lingüísticos. $\mathrm{n}^{\mathrm{O}} 36$, pp. 125-165.

FABRÍCIO, B. F. (2013). A "outridade lusófona" em tempos de globalização: identidade cultural como potencial semiótico. En: Lopes, L. P. da Moita (org.), O português no século XXI. Cenário geopolítico e sociolinguístico. San Pablo: Parábola, pp. 144-168.

FARACO, C. A. (2010). A Encruzilhada da lusofonia. Grial, v. 48, nº 187, pp. 76-81.

FARACO, C. A. (2012). Lusofonia: utopia o quimera? Língua, história e política. En: Lobo, T.; Carneiro, Z.; Soledade, J.; Almeida, A.; Ribeiro, S. (orgs.), Rosae: linguística bistórica, bistória das línguas e outras bistórias. Salvador: EDUFBA, pp. 31-50.

FARACO, C. A. (2016). História sociopolítica da língua portuguesa. San Pablo: Parábola.

FIORIN, J. L. (2009). O acordo ortográfico: uma questão de política linguística. Veredas, v. 24, pp. 7-19.

FIORIN, J. L. (2010). Língua portuguesa, identidade nacional e lusofonia. En: Bastos, N. B. (org.), Lingua portuguesa: cultura e identidade nacional. San Pablo: EDUC, pp. 15-30.

FIORIN, J. L. (2013). Língua, identidades e fronteiras. Diversitas, nº 1, pp. 147-164.

GOMES, N. L.; VIEIRA, S. L. (2013). Construindo uma ponte Brasil-África: a Universidade da Integração Internacional da Lusofonia Luso-Afrobrasileira (UNILAB). Revista Lusófona de Educação, nº 24, pp. 75-88.

HANNA, V. L.; BRITO, R. P.; BASTOS, N. B. (2010). Políticas de língua e lusofonia: aspectos culturais e ideológicos. En: Martins, M. L.; Cabecinhas, R.; Macedo, L. 
(eds.), Anuário Internacional de comunicação lusófona. Lusofonia e sociedade em rede. Braga: Grácio Editor, pp. 159-175.

LAGARES, X. C. (2013). O espaço político da língua espanhola no mundo. Trabalhos em Linguística Aplicada, v. 52, n 2, pp. 385-408.

LAGARES, X. C. (2016). Dinámicas normativas del español y del portugués. En: Arnoux, E. N. de; Lauria, D. (comps.), Lenguas y discursos en la construcción de la ciudadanía sudamericana. Gonnet: UNIPE, pp. 283-297.

LAGARES, X. C. (2018). Dinâmicas normativas e autoridade linguística em espanhol e português: uma gestão da ortografia. En: Sousa, S. C. T.; Roca, M. P.; Ponte, A. (orgs.), Temas de política linguística no processo de integração regional. Campinas: Pontes, pp. $175-198$.

LEVISKI, C. (2015). A CPLP e a virada econômica: Guiné Equatorial em foco. En: Encuentro Internacional de Investigadores de Políticas Lingüísticas, $\mathrm{n}^{\circ}$ 7. Córdoba: Facultad de Lenguas, Universidad Nacional de Córdoba, v. 1. pp. 245-252.

LOPES, L. P. da Moita (2013). Ideologia linguística: como construir discursivamente o português no século XXI. En: Lopes, L. P. da Moita (org.), O português no século XXI. Cenário geopolítico e sociolinguístico. San Pablo: Parábola, pp. 18-52.

MARTINS, M. L. (2006). A lusofonia como promessa e o seu equívoco lusocêntrico. En: Martins, M. L.; Cabecinhas, H. S. (eds.), Comunicaçao e lusofonia. Para uma abordagem crítica da cultura e dos media no espaço lusófono. Porto: Campo das Letras, pp. 79-87.

MARTINS, M. L. (2014). Língua portuguesa, globaliçazão e lusofonia. En: Bastos, N. (org.), Lingua Portuguesa e Lusofonia. San pablo: EDUC, pp. 15-33.

MARTINS, M. L. (coord.) (2015). Lusofonia e Interculturalidade. Promessa e travessia. V. N. Famalicão: Húmus.

OLIVEIRA, G. Müller de (2013a). Um Atlântico ampliado: o português nas políticas linguísticas do século XXI. En: Lopes, L. P. da Moita (org.), O português no século XXI. Cenário geopolítico e sociolinguístico. San Pablo: Parábola, pp. 53-73.

OLIVEIRA, G. Müller de (2013b). Política linguística e internacionalização: a língua portuguesa no mundo globalizado do século XXI. Trabalhos em Linguística Aplicada. v. ${ }^{\circ}$ $52, \mathrm{n}^{\circ} 2$, pp. 409-433. 
OLIVEIRA, G. Müller de (2015). O Instituto Internacional da Língua Portuguesa e a gestao multilateral da Língua Portuguesa no âmbito da CPLP. Revista Internacional de Linguística Iberoamericana. v. XIII, n ${ }^{\circ} 1$ (25), pp. 19-34.

OLIVEIRA, G. Müller de; DORNELLES, C. (2007). Políticas internacionales del portugués. $A R I, n^{\circ} 135$, Real Instituto Elcano. Disponible en: http://www.realinstitutoelcano. org/analisis/ARI2007/ARI135-2007 Muller Dornelles.pdf. Acceso: 15 jun. 2018.

ORLANDI, E. P. (2009). Língua Brasileira e outras bistorias. Discurso sobre a língua e ensino no Brasil. Campinas: Editora RG.

ORLANDI, E. P. (2012). Espaços linguísticos e seus desafios: convergencias e divergencias. Revista Rua, v. 2, n 18. Disponible en: http://www.labeurb.unicamp.br/rua. Acceso: 15 jun. 2018.

PINTO, J. F. (2011). Da CPLP à Comunidade Lusófona: o futuro da lusofonia. Revista Angolana de Lusofonia. nº 7, pp. 107-118.

REIS, C. (2010). La internacionalización de la lengua portuguesa. Contextos, confrontaciones y prioridades. En Ciclo de conferencias 2010 "El espacio ibérico de las lenguas". Madrid: Instituto Cervantes. Disponible en: https://www.cervantes.es/imagenes/file/lengua/jornadas/ carlos reis 2010ic.pdf. Acceso: 15 jun. 2018.

REIS, C. (2015). Espaços da língua portuguesa ou os perigos da imagináutica. En: Ferreira, M. A.; Brasete, M. F. (eds.), Pelos Mares da Lingua Portuguesa 2. Aveiro: Univ. de Aveiro, pp. 9-20.

RIZZO, M. F. (2014). Antecedentes de la política lingüística panhispánica: los Congresos Internacionales de la Lengua Española. En: Arnoux, E. N. de y S. Nothstein (eds.), Temas de Glotopolítica: integración regional y panbispanismo. Buenos Aires: Biblos, pp. 191 220.

SEVERO, C. G. (2015). A açucarada língua portuguesa: Lusotropicalismo e Lusofonia no século XXI. Revista Brasileira de Linguistica Aplicada, v. 15, pp. 85-107.

SEVERO, C. G. (2016). Lusofonia, Colonialismo e Globalização. Fórum Linguistico (Online), v. 13, pp. 1321-1333.

SIGNORINI, I. (2013). Política, lengua portuguesa e globalição. En: Lopes, L. P. da Moita (org.), O português no século XXI. Cenário geopolítico e sociolinguístico. San Pablo: Parábola, pp. 74-100. 
SILVA, D. B. (2010a). A Promoção da Língua Portuguesa nos Discursos Oficiais do Ministério da Cultura do Brasil. Linguasagem. v. 14, pp. 1-19.

SILVA, D. B. (2010b). O passado no presente: história da promoção e difusão da Língua Portuguesa no exterior. Cadernos do CNLF. v. 14, n . 4, t.4, pp. 3018-3034.

SILVA, D. B. (2011). Ortografia unificada: arena de disputas entre Portugal e Brasil. Palimpsesto, v. 13, pp. 1-35.

SILVA, D. B. (2012). As contradições da cooperação técnica em educação Brasil-CPLP: o caso do Timor-Leste. Carta Internacional (USP), v. 7, pp. 127-148.

SILVA, D. B. (2013). De flor do Lácio a língua global. Jundiaí: Paco Editorial.

SILVA, D. B.; SANT’ANNA, V. (2012). Fabrica-se um "novo portugués"? Uma análise discursiva de documentos da CPLP. Gragoatá. v. 32, pp. 117-136.

ZOPPI-FONTANA, M. G. (org.) (2009). O Português do Brasil como Língua Transacional. Campinas: Editora RG.

ZOPPI-FONTANA, M. G. (2010). Ser brasileiro no mundo globalizado: alargando as fronteiras da língua nacional. En: Di Renzo, A. M. et al. (orgs.), Linguagem e História. Múltiplos Territórios Teóricos. Campinas: Editora RG, pp. 129-152.

ZOPPI-FONTANA, M. G. (2012). A arte do detalhe. Web Revista Discursividade, Estudos Linguísticos. $n^{\circ}$ 9. Disponible en: http://www.discursividade.cepad.net.br/. Acceso: 15 jun. 2018.

Recebido: 26/12/2018

Aceito: $7 / 02 / 2019$

Publicado: 29/03/2019 